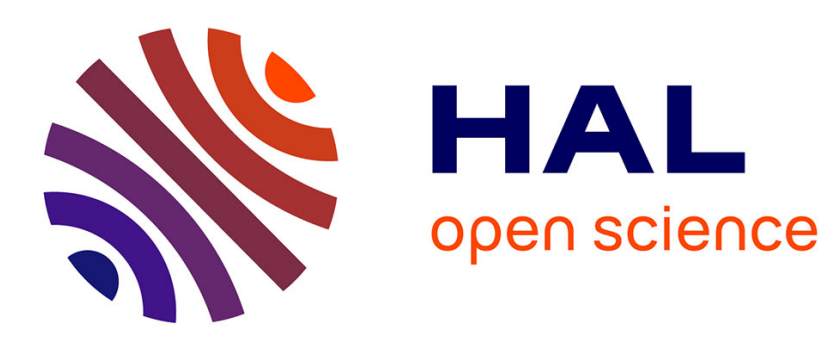

\title{
Les catégories d'odeurs en sont-elles vraiment ?
}

Joël Candau, Olivier Wathelet

\section{To cite this version:}

Joël Candau, Olivier Wathelet. Les catégories d'odeurs en sont-elles vraiment ?. Langages, 2011, 181

(1), 10.3917/lang.181.0037 . halshs-01723678

\section{HAL Id: halshs-01723678 \\ https://shs.hal.science/halshs-01723678}

Submitted on 5 Mar 2018

HAL is a multi-disciplinary open access archive for the deposit and dissemination of scientific research documents, whether they are published or not. The documents may come from teaching and research institutions in France or abroad, or from public or private research centers.
L'archive ouverte pluridisciplinaire HAL, est destinée au dépôt et à la diffusion de documents scientifiques de niveau recherche, publiés ou non, émanant des établissements d'enseignement et de recherche français ou étrangers, des laboratoires publics ou privés. 


\title{
LES CATÉGORIES D'ODEURS EN SONT-ELLES VRAIMENT?
}

Joël Candau, Olivier Wathelet

\author{
Armand Colin | « Langages »
}

$2011 / 1 n^{\circ} 181 \mid$ pages 37 à 52

ISSN 0458-726X

ISBN 9782200926809

Article disponible en ligne à l'adresse :

http://www.cairn.info/revue-langages-2011-1-page-37.htm

\section{Pour citer cet article :}

Joël Candau, Olivier Wathelet« Les catégories d'odeurs en sont-elles vraiment ? », Langages 2011/1 ( $\left.{ }^{\circ} 181\right)$, p. 37-52.

DOI 10.3917/lang.181.0037

Distribution électronique Cairn.info pour Armand Colin.

(C) Armand Colin. Tous droits réservés pour tous pays.

La reproduction ou représentation de cet article, notamment par photocopie, n'est autorisée que dans les limites des conditions générales d'utilisation du site ou, le cas échéant, des conditions générales de la licence souscrite par votre établissement. Toute autre reproduction ou représentation, en tout ou partie, sous quelque forme et de quelque manière que ce soit, est interdite sauf accord préalable et écrit de l'éditeur, en dehors des cas prévus par la législation en vigueur en France. Il est précisé que son stockage dans une base de données est également interdit. 
Joël Candau

Université de Nice Sophia Antipolis \& Laboratoire d'Anthropologie et de Sociologie

'Mémoire, Identité et Cognition Sociale' (LASMIC, EA 3179)

\section{Olivier Wathelet}

Université de Nice Sophia Antipolis \& Laboratoire d'Anthropologie et de Sociologie

'Mémoire, Identité et Cognition Sociale' (LASMIC, EA 3179)

\section{Les catégories d'odeurs en sont-elles vraiment ?}

\section{INTRODUCTION}

C. Lévi-Strauss associait le Brésil à une « odeur de cassolette » en raison de « l'homophonie inconsciemment perçue des mots <Brésil > et <grésiller>» (1955:50). Une bonne tomate, nous a dit un jour un grand chef, sent "l'élégance, la vérité, l'honnêteté » ${ }^{1}$ (Candau, 2000 : 75). Lors de mes visites à Combray, écrit le narrateur, « je revenais toujours avec une convoitise inavouée m'engluer dans l'odeur médiane, poisseuse, fade, indigeste et fruitée du couvre-lit à fleurs » de tante Léonie (Proust, 1987 : 61). Selon un de nos informateurs, fossoyeur, le corps d'une personne noyée qui a séjourné longtemps dans l'eau dégage des " odeurs à ne pas regarder » (Candau \& Jeanjean 2006). Au cours d'une expérience de reconnaissance d'odeurs conduite dans notre université (Baccino et al. 2010), plusieurs participants ont décrit l'odeur de substances chimiques pures en termes parfois étonnants : "parmesan râpé, un peu vieux", " odeur de vieux métal ", de " torchon mouillé », "la pluie, qui est tombée par terre, quand il y a un peu le chaud et tout...", "ça sent comme un réveil au matin", "les vers noirs de pluie ", odeur "qui se rapproche de l'amour ", etc. Si on en juge par ces quelques exemples de dénomination ou de désignation des odeurs, la diversité de leur description ne semble bornée que par l'étendue du langage maîtrisé par le locuteur. Dès lors se pose la

\footnotetext{
1. Tout au long du texte, nous distinguons par l'emploi de l'italique les extraits d'entretiens des autres données ethnographiques. Ce sont ainsi deux types de discours que nous avons récoltés et analysés. Le premier, en italique, est fait de verbalisations a posteriori suscitées en contexte d'entretiens semi-directifs pour décrire et expliquer des expériences et des expertises olfactives en milieu professionnel et domestique (voir, en particulier, Candau 2000). Le second consiste en anecdotes olfactives extraites de forums de discussions en ligne. Non suscitées par le chercheur, ces anecdotes n'ont pas le caractère systématique et cohérent des données d'entretiens. Elles rendent plus difficile l'identification de leur auteur, mais permettent, grâce à leur nombre, une description minutieuse des jugements olfactifs, et favorisent la constitution d'un large corpus de dialogues concernant un thème donné (voir Wathelet, 2009 : chap. 2 pour une discussion de la méthode).
} 
question des modalités de leur catégorisation. Dans quelle mesure le caractère foisonnant et, en apparence, instable et imprécis du langage naturel des odeurs i.e. le langage ordinaire que nous utilisons dans la vie quotidienne pour évoquer nos expériences olfactives - entrave-t-il ou favorise-t-il la construction de catégories perceptives et sémantiques qui sont supposées mettre de l'ordre dans le chaos des stimuli ? Plus généralement, quelles sont les caractéristiques de ce processus de catégorisation appliqué aux expériences olfactives?

Après un rappel de la théorie de la catégorisation aujourd'hui dominante en sciences cognitives, nous examinerons la pertinence de son application dans le registre du sensible ou, plus exactement, dans la manière dont la catégorisation des expériences sensibles est exprimée en langue. Nous soutiendrons que, si la catégorisation des couleurs s'accorde assez bien avec cette théorie, les données ethnographiques que nous avons recueillies dans le domaine des odeurs en contexte de langage naturel ${ }^{2}$ ruinent l'idée selon laquelle les "catégories » olfactives sont ce qu'elles prétendent être : elles ne sont pas des catégories $\mathrm{du}$ point de vue de la théorie classique et encore moins du point de vue de la théorie des prototypes, hégémonique depuis les années 70 . Cependant, les stimuli olfactifs sont l'objet de jugements perceptifs qui permettent de construire des pseudo-prototypes, i.e. qui se donnent à voir dans le langage comme des prototypes alors qu'ils n'en sont pas du strict point de vue de leur traitement cognitif. Nous conclurons par quelques considérations générales sur les causes possibles de ce « relâchement » catégoriel.

\section{LES THÉORIES DE LA CATÉGORISATION}

Il est admis que « la catégorisation est principalement un moyen de comprendre le monde » (Lakoff \& Johnson, 1985 : 132). En effet, notre perception des choses ne s'arrête jamais aux étants singuliers tels que les définit la philosophie ockamienne. Du fait de nos dispositions innées à l'abstraction (Quian Quiroga et al. 2005), chaque événement est aussitôt traité comme membre d'une catégorie qui en véhicule le concept. Par exemple, depuis le bureau où nous sommes en ce moment, nous entendons la pluie tomber, péripétie météorologique que nous interprétons comme l'occurrence d'un phénomène général, celui de « la pluie », catégorie abstraite qui est censée rendre compte de tous les épisodes pluvieux, passés, présents et futurs connus par notre planète et même par l'univers tout entier. Tous ces épisodes ont en commun des attributs qui obéissent à une combinatoire - la pluie est constituée de gouttes d'eau, ces gouttes tombent du ciel, elles viennent de nuages, elles mouillent le sol, etc. (Searle 2004) - et qui sont

\footnotetext{
2. Notre démarche se veut complémentaire des approches lexicographiques comparatives qui font émerger des régularités inter-langagières dans le traitement des informations olfactives comme, par exemple, l'étude canonique de Boisson (1997). Les conclusions de l'auteur - un fort relâchement catégoriel inter-langagier nous paraissent congruentes avec notre propos.
} 
subsumés dans la catégorie " pluie », elle-même enchâssée dans d'autres catégories constituant des schemata ou des systèmes de croyances. Cette "opération cognitive » (Bradford \& Caramazza 2009) est probablement un trait adaptatif de notre espèce qui nous a permis une discrimination naturelle (Peelen, Fei-Fei \& Kastner 2009) d'unités d'information importantes dans notre environnement (êtres animés vs choses inanimées ; proies vs prédateurs ; jour vs nuit, etc.), compétence que nous utilisons par ailleurs culturellement de manière massive (catégoriser des outils lithiques, des genres littéraires, des timbres-poste, des véhicules à moteur, etc.). Mais comment construisons-nous ces catégories ?

Selon l'approche classique (dite aristotélicienne), les catégories sont des entités aux frontières nettes, représentées par un ensemble de conditions nécessaires et suffisantes. La pertinence de la catégorisation est reconnue en évaluant si toutes les conditions d'entrée dans la catégorie sont satisfaites. Par exemple, si l'objet qui est devant nous a un siège, un dossier et quatre pieds, alors c'est une chaise, et nous pouvons le ranger dans la catégorie "chaises ». Aujourd'hui, cette approche classique n'est plus jugée satisfaisante par les théoriciens de la catégorisation car elle occulte un fait essentiel : dans une catégorie, certains membres sont psychologiquement plus saillants et plus faciles à mémoriser. À ce titre, ils sont jugés meilleurs exemplaires de cette catégorie. Par exemple, un canari est considéré comme un meilleur exemplaire de la catégorie " oiseaux » qu'un manchot ou une autruche. De même, un chien ou un éléphant sont jugés meilleurs exemplaires de la catégorie " animaux » que le corail. Voilà pourquoi, suite aux travaux de la psychologue E. Rosch, une autre théorie de la catégorisation a supplanté l'approche classique. Le phénomène pris en compte est celui de la "typicalité » : certains exemplaires sont particulièrement typiques de la catégorie et l'un d'entre eux a un caractère de prototypicalité. Les prototypes, écrit E. Rosch, sont « les tendances centrales des catégories » (Rosch 1973). Autrement dit, le prototype est l'exemplaire le plus représentatif de la catégorie et, à ce titre, il est un point de référence cognitif pour la conscience perceptive.

La théorie roschienne s'est nourrie des recherches menées dans les années 60 par les anthropologues B. Berlin et P. Kay (1991) sur la perception des couleurs. Leur thèse peut se résumer simplement : il existe des couleurs élémentaires (noir, blanc, rouge, jaune, vert, bleu, brun, violet, rose, orange et gris) qui sont des " universaux » et des termes désignant les catégories de ces couleurs élémentaires (Basic Color Terms). Selon les cultures, ces termes ne correspondent pas nécessairement au nombre de couleurs perçues et leur présence semble obéir à une règle hiérarchique : par exemple, on ne peut pas avoir le rose ou l'orange si on n'a pas le rouge ou le jaune. Par ailleurs, si les frontières des catégories fondamentales peuvent varier d'une culture à l'autre, on obtient un consensus panhumain lorsque l'on demande à des individus de désigner sur un nuancier les meilleurs exemplaires des couleurs élémentaires. Ainsi, dans la catégorie " rouge », le même point focal sera partout désigné comme le meilleur représentant de la couleur. En bref, soutient E. Rosch "some reds are redder than others" (1975 : 198). 
Outre la notion de prototype, qui est au cœur de la théorie roschienne, $\mathrm{d}^{\prime}$ autres règles et principes caractérisent celle-ci ${ }^{3}$. L'un d'entre eux, le principe d'économie cognitive, est considéré comme au fondement de la catégorisation, quelle que soit la théorie proposée pour cette opération mentale. Ce principe revient à pondérer deux orientations cognitives opposées : la première visant à multiplier les catégories pour maximiser l'information portée par les stimuli, orientation qui, poussée à son extrême, conduit à avoir autant de catégories que d'objets différents rencontrés dans le monde ; la seconde orientation consistant à limiter le nombre de catégories de telle sorte que leur utilisation représente une charge cognitive supportable. La catégorisation autour du prototype est sans doute un compromis entre ces deux orientations. Le prototype, qui en est le point d'équilibre et que l'on peut assimiler à un condensateur de l'information portée par les différents membres, remplit de ce fait une fonction d'allègement du fardeau cognitif.

Un autre principe est également central dans la théorie prototypique : l'organisation des catégories selon deux dimensions, l'une horizontale et l'autre verticale. La dimension verticale fait référence à un rapport d'hyponymie entre différents niveaux de catégorisation. Le niveau super-ordonné (e.g. " animal ») inclut un niveau de base (e.g. " chat») qui inclut un niveau subordonné (e.g. « chat persan »). On retrouve le même type d'inclusion entre animal/oiseau/rossignol et animal/chien/caniche. La dimension horizontale fait référence aux concepts placés au même niveau d'inclusion. Il y a entre eux une relation sémantique horizontale, comme c'est le cas entre chat persan, rossignol et caniche. Le niveau de base et le niveau subordonné s'opposent au niveau super-ordonné en ce que les membres de leurs catégories sont perçus comme ayant une forme (Gestalt) semblable. Il n'y a pas, en effet, de forme générale qui corresponde à " animal » alors que l'on en perçoit une pour « chien » et, bien entendu, pour " caniche ». Le niveau de base est, en conséquence, le niveau le plus élevé (le plus abstrait) où les membres des catégories ont des formes globales perçues de façon similaire et où, du même coup, les catégories entre elles sont le mieux différenciées. Pour cette raison, il est réputé être le niveau d'entrée privilégié dans la catégorie, même si cela semble moins vrai pour des membres atypiques de celle-ci (Murphy \& Brownell 1985) ou dans le cas de la catégorisation visuelle (Macé et al. 2009). Il représente donc un optimum du point de vue de l'efficacité cognitive de la catégorisation.

Ce niveau de base est également celui où les individus d'une espèce donnée perçoivent dans les membres de la catégorie la même affordance. Celle-ci peut être définie comme la possibilité d'action qu'offre un objet par sa pure matérialité, au regard du système perceptif de cette espèce (Gibson 1979). Pour des êtres vivants ayant une conformation humaine, une chaise induit l'action de s'asseoir, de la même manière que certains galets sur la plage induisent l'action de faire

3. Sans le citer à chaque fois, nous prendrons souvent appui sur l'analyse que propose Kleiber (1991) de la théorie roschienne. 
des ricochets. Cela signifie qu'au niveau de base, les membres de la catégorie déclenchent un programme moteur similaire chez les individus qui interagissent avec eux. Par exemple, un lit induira l'action particulière de s'allonger, ce que ne peut pas faire la catégorie super-ordonnée de "mobilier».

Un autre principe est celui de l'exploitation de la structure du monde perçu. Il est fondé sur l'idée que, dans notre environnement, certaines combinaisons $\mathrm{d}^{\prime}$ attributs sont plus fréquentes que d'autres. Par exemple, s'il est très courant de rencontrer une créature ailée qui a des plumes et un bec, il est beaucoup moins probable de rencontrer un mammifère doté de ce dernier attribut et qui pond des œufs. Si, en disant cela, nous pensons immédiatement à l'ornithorynque, c'est précisément à cause de son caractère d'exceptionnalité. On touche là à la notion de cue validity, qui est le degré de prédictibilité d'une propriété pour une catégorie. La cue validity de la propriété " avoir des plumes » est élevée pour la catégorie " oiseau » bien que nous puissions observer des oiseaux sans plumes (dans nos assiettes) et des êtres vivants avec des plumes qui ne sont pas des oiseaux (par exemple, des chefs indiens). Selon E. Rosch, l'exploitation de cette structure relationnelle préside à l'élaboration de nombreuses catégories.

Enfin, les termes utilisés au niveau de base ont plusieurs caractéristiques. Ils obéissent généralement à un principe de parcimonie linguistique : on utilisera « jaune » et non « jaune canari » ou « vert » et non « vert amande ». Ils sont appris plus tôt que d'autres par les enfants et ce sont les mots que des adultes utilisent spontanément, le plus souvent quand ils doivent dénommer librement des objets. Ils se situent, par ailleurs, au niveau de dénomination le plus générique, ce que l'on vérifie aisément avec les couleurs élémentaires : si « vert amande » fait partie de la catégorie " vert », l'inverse n'est évidemment pas vrai.

Aujourd'hui, et malgré un retournement critique de son auteur au début des années 90 (Varela, Thompson \& Rosch 1993), les thèses roschiennes continuent à peser fortement dans les débats sur la catégorisation des couleurs, qu'il s'agisse de prolonger les travaux de B. Berlin et P. Kay ou d'essayer de les récuser (Davidoff 2001 ; Davidoff, Davies \& Roberson 1999 ; Wierzbicka 2008). Nous n'avons pas la place, ici, de développer ces questions. Notons simplement que, même si elle est régulièrement ébranlée, la théorie roschienne reste robuste. Des travaux les plus récents sur la perception et la catégorisation des couleurs (Kay \& Regier 2003, 2006, 2007 ; Regier, Kay \& Khetarpal 2007 ; Regier, Kay \& Cook 2005) se dégagent les conclusions suivantes : les différences observées dans la dénomination des couleurs ont des effets sur leur cognition (notamment la mémoire des couleurs et leur discrimination); ces effets sont renforcés et peuvent même jouer sur la catégorisation (Zou et al. 2010) lorsque les couleurs sont présentées dans le champ visuel droit (et donc traitées par l'hémisphère gauche), mais il n'en reste pas moins qu'à ce jour on n'a pas infirmé la thèse de l'existence de contraintes universelles sur la catégorisation des couleurs élémentaires, autour d'un point focal pour chacune d'entre elles. 
Bref, même si la théorie des prototypes affronte des concurrents sérieux (Barsalou 1983, 1985) et même si, pas plus qu'une autre théorie, elle est loin d'être parfaite, nous disposons cependant avec elle d'un ensemble de concepts (prototype, économie cognitive, dimensions verticale et horizontale, structure du monde perçu, parcimonie linguistique) qui permettent de rendre compte de manière satisfaisante de la construction des catégories à partir d'une grande variété de stimuli venant du monde physique ou social. Reste-t-elle opératoire dans ce registre si particulier du sensible qu'est l'olfaction?

\section{LES CATÉGORIES D'ODEURS À L'ÉPREUVE DE LA THÉORIE ROSCHIENNE}

Les données ethnographiques recueillies en contexte professionnel et domestique révèlent des compétences classificatoires diverses, en fonction de l'intérêt des informateurs pour la matière odorante. Quand il s'agit de traiter les informations mondaines, des pratiques spécifiques sollicitent massivement l'usage de l'odorat et, comme on peut s'y attendre, certains experts professionnels (œnologues, chefs de cuisine, parfumeurs) et domestiques (dans le domaine de la cuisine, de l'esthétique et de l'hygiène) recourent à un vocabulaire olfactif plus étendu. Pour autant, observe-t-on l'émergence de catégories olfactives satisfaisant aux principes qui gouvernent la théorie roschienne de la catégorisation? Reprenons les un par un.

\subsection{Prototypicalité}

Dans nos enquêtes ethnographiques, comme dans les expériences que nous avons montées au sein du groupe MOD (Molécules, Olfaction, Discours) de l'Université de Nice-Sophia Antipolis, les informateurs et les participants ont souvent catégorisé les stimuli olfactifs sur la base d'un air de famille ${ }^{4}$. Un œnologue, par exemple, évoquera " des odeurs qui sont un peu de la même famille que les odeurs de bouchon » ou " des odeurs de la famille d'un oignon » ou encore « de la famille de l'ail ». Cependant, cet air de famille ne semble pas pouvoir être rattaché à une odeur fondamentale, comme c'est le cas pour les couleurs. Il renvoie plutôt à des stratégies locales de description, plus ou moins partagées au sein d'une même communauté d'intérêts. Ainsi, quand une cuisinière sur un forum Internet (Wathelet 2009) cherche à retrouver un ingrédient dont elle ne garde que le souvenir, elle avance avec assurance la description suivante : "C'était des cubes-bouillon (enfin pas cubes plutôt forme de pastille ovale) très foncés genre Viandox niveau goût et odeur ». Au-delà de ces formes locales que 
l'on peut expliquer par des dynamiques culturelles ${ }^{5}$, rien ne nous autorise à faire l'hypothèse de l'existence d'odeurs primaires ou " focales ». Considérons, par exemple, la catégorie « odeurs florales ». Que pourrait être l'odeur focale ou la tendance centrale, ou le meilleur exemplaire - de cette catégorie ? Le jasmin ? La rose ? La pervenche ? On doute qu'il y ait une réponse universelle à cette question. Certes, telle ou telle odeur de fleur peut avoir une saillance particulière. Par exemple, l'odeur de jasmin est bien plus saillante que celle d'une tulipe. Ce phénomène, note A. Holley (1999), peut être provoqué par le mode de fonctionnement de notre système neurosensoriel ou dépendre fortement de l'environnement culturel. Cependant, il semble bien que l'on ne puisse parler $\mathrm{d}^{\prime}$ " odeurs fondamentales ». Comme l'observe D. Valentin (c.p.), la base de la catégorisation des odeurs se révèle amplement fonctionnelle, répondant à des critères de comestibilité, de dangerosité ou encore, à des attributs d'hédonicité et d'utilité. Dans cette perspective utilitariste, un producteur d'huile d'olive de la région de Nice explique en toute logique que "l'odeur du tonneau, c'est bon, ce n'est pas bon; il y a un problème, il n'y a pas de problème ». L'accumulation, dans nos entretiens, de verbatim similaires à celui-ci souligne la prégnance des conditions d'usage lors de la catégorisation des odeurs.

Par ailleurs, dans le registre de l'olfaction, il y a toujours une incertitude - du point de vue de l'anthropologue - à propos de ce qui est réellement catégorisé. En effet, on observe communément un recouvrement des référents du terme " odeur » par l'objet odorant. L'odeur, qui est la représentation cognitive de l'odorant, est la plupart du temps confondue avec l'odorant lui-même, ce qui ajoute à l'imprécision de la description. Ce que l'on appelle l'« odeur de rose ", par exemple, est en réalité l'effet que fait l'odorant " rose » chez un sujet mis en sa présence. Dans ce cas, alors que le descripteur olfactif renvoie explicitement à la source odorante considérée, par ailleurs et à tort, comme équivalente à la nature objective de la substance odorante - il serait en effet plus exact, toujours du point de vue de l'anthropologue, de parler de "l'odeur des molécules odorantes de la rose »-, il décrit la qualité perçue, c'est-à-dire subjective, de cette substance.

Or, dans le langage ordinaire, nous sommes régulièrement confrontés à ces " confusions ». L'odeur de rose n'est pas la même quand elle est conceptualisée par un informateur en termes d'effet ou comme l'indice d'un événement porteur de sens. Dans le premier cas, elle est catégorisée selon des critères quasi physiologiques : "une odeur de rose entêtante ». Dans le second, elle est catégorisée au regard des dimensions pertinentes dans le contexte de la perception. L'odeur de rose peut appartenir alors à la catégorie " odeur de fleur " pour une mère ou un père promenant ses enfants dans un jardin et peut devenir une " odeur de fille » pour un jeune homme discutant des parfums de sa compagne; deux contextes de catégorisation de cette odeur que nous avons observés. Dans ces exemples, la

5. Le Viandox est un produit caractéristique de l'industrie agroalimentaire du début du XX $\mathrm{XX}^{\mathrm{e}}$ siècle, dont le rôle de référent sensoriel est lié à l'histoire de sa diffusion au sein des cuisines domestiques. 
façon dont est implicitement articulé le trio odeur/odorant/source de l'odeur, conditionne la catégorisation de la perception.

Enfin, alors que dans le domaine des couleurs les rouges " plus rouges que les autres ", selon les termes d'E. Rosch, le restent quel que soit le contexte, il n'en va pas de même avec les odeurs dont la catégorisation est sous l'emprise d'effets contextuels (écologiques). Par exemple, l'odeur du Munster, catégorisée comme une odeur alimentaire ( « de fromage ») à la fin d'un repas, sera catégorisée comme une mauvaise odeur si elle est perçue en entrant chez un fleuriste. Même si on sait qu'en jouant de certaines variables contextuelles, on peut modifier la perception des couleurs, celle-ci n'est pas aussi dépendante du contexte que les odeurs. Dans le contexte de techniques professionnelles ou domestiques impliquant une certaine répétition de l'activité, le lien avec le contexte est l'élément qui sert à catégoriser celles-ci. Ainsi, à l'occasion d'une pratique culinaire consistant à faire passer des ingrédients du cru au cuit, les odeurs sont catégorisées selon leur chronologie dans le processus de cuisson. Les conseils donnés sur des forums culinaires l'illustrent assez bien :

[Riz basmati cuit dans de l'huile de cumin chauffée] Quand une bonne odeur se développe, ajouter le riz, faire revenir. (une femme, sans autre identification)

[Une recette réunionnaise] Une fois ceci fait... tu devrais avoir une bonne odeur de cari dans les narines... (un homme, âge inconnu, originaire de La Réunion)

Faire griller les feuilles de nori ${ }^{6} \mathrm{~d}^{\prime}$ un côté, jusqu'à ce qu'elles commencent à dégager une bonne odeur. (un homme, âge inconnu, originaire de Thailande)

Il ressort de ces exemples que l'identification de l'odeur ne repose pas sur une catégorisation absolue, dont témoignerait un descripteur spécifique, mais sur le moment de son apparition au sein d'une séquence d'événements significatifs pour le cuisinier, que nous proposons d'appeler un " cheminement perceptif ». De ce fait, dans ces situations de la vie quotidienne, l'attribution d'une odeur à une catégorie stabilisée sous une forme indépendante du contexte de perception est rarement nécessaire à l'efficacité de l'action et de sa communication :

[Du beurre noisette pour mélanger avec des blancs d'cuf] Fondre le beurre dans une casserole à feu vif jusqu'a ce que vous sentiez une odeur de noisette. (une femme, 26 ans, Bastia)

Au contraire, dans cet exemple comme dans les précédents, c'est la place dans une trame perceptive - tout comme le repas est décomposable en unités olfactives distinctes selon les cultures (Candau 2002) - qui fonde la catégorisation. Le contexte est ici une dimension indispensable à l'opération de catégorisation, car il définit l'utilité de la perception au regard de la tâche en cours (permettre et contrôler une transformation d'une matière alimentaire). L'hyperdépendance de ce processus aux effets contextuels et à des séquences d'événements perceptifs rend très improbable l'identification d'odeurs ayant la qualité de prototypes qui, par définition, requièrent une certaine stabilité, décontextualisée sommes-nous

6. La feuille de nori est l'ingrédient qui recouvre le riz lors de la préparation des sushis. 
tentés de dire. Au sens strict du terme, il paraît donc très hasardeux de parler d'odeurs prototypiques.

\section{2. Économie cognitive}

L'hyperdépendance contextuelle de la perception olfactive n'est pas sans conséquence sur un autre principe essentiel de la catégorisation, celui d'économie cognitive. Dans le cas des odeurs, il est soit complètement absent, soit pèche par excès. En effet, parce que la catégorisation des odeurs est instable et très vulnérable au contexte, elle se manifeste soit par une profusion catégorielle, soit par la réduction drastique de l'information à un tout petit nombre de catégories très générales. La profusion a pour cause principale l'identification courante d'une odeur à sa source. Le locuteur prend alors appui non seulement sur des catégories très larges telles que " odeurs florales ", " animales ", " fruitées ", etc., mais aussi sur d'innombrables catégories spécifiques telles que odeur de rose, de lilas, de chien mouillé, de fauve, de citron, de banane, etc. En définitive, on obtient presque autant de catégories que de sources.

Mais, on observe tout aussi bien des processus de catégorisation extrêmement frustes - ce qui ne veut pas dire inefficaces -, dès lors que la référence à la source est abandonnée au profit d'une description davantage phénoménologique, en termes de quale ou " effet que cela fait" (Nagel 1974). On obtient alors des catégories sommaires et binaires, jouant des oppositions canoniques " agréable vs désagréable » (critère hédonique), "fort vs faible » (critère de l'intensité), " connu vs inconnu » (critère de la familiarité). Dans ce cas, l'économie cognitive est incontestable, mais le prix à payer est un appauvrissement de l'information, du moins d'un point de vue extérieur à l'expérience olfactive, car la plupart du temps peut-être suffit-il au sujet percevant de savoir qu'une odeur est « bonne » ou « mauvaise », ou connue ou inconnue.

L'analyse de nos données dévoile un troisième mécanisme, qui est l'extension progressive du contenu catégoriel au fil des situations de perception. Il se manifeste, en particulier, en présence des odeurs du corps. Les parures cosmétiques tendent progressivement à être intégrées au sein de la catégorie " odeur de + individu particulier/une catégorie particulière d'individu », comme cette maman qui sent un vêtement pour retrouver l'odeur de ses enfants : "Quand elles sont au lit et qu'elles me manquent, je respire fort leur odeur dans les bodies : rien de mieux que l'odeur de la peau d'un bébé » (une femme, 27 ans, originaire des USA). Ce processus d'extension progressive peut être si fort que les propositions catégorielles prennent une forme (apparemment) tautologique, du type : «j'aime qu'un bébé sente le bébé » (une femme, 30 ans, Marne).

\subsection{Les dimensions verticale et horizontale}

La hiérarchie catégorielle des odeurs est incertaine, tout au moins dans son expression linguistique. Le type et l'exemplaire sont mal distingués : il est fréquent que les mêmes dénominations soient proposées dans les réponses 
«type » et les réponses « exemplaire » (David, $1997: 213)$. Au contraire, dans le domaine des couleurs, il est aisé d'aller du plus abstrait vers le moins abstrait, ou du moins spécifique vers le plus spécifique, comme par exemple dans le cas suivant : coloré>rouge>vermillon. On a ici une lignée sémantique avec des relations d'implication entre propriétés : un objet qualifié de vermillon implique qu'il soit considéré comme rouge, s'il est considéré comme rouge, cela implique qu'il ait une couleur (Chauveix \& Egoroff, 2003 : 83). Cette implication des propriétés qui rend possible ces inférences est-elle présente lors de la catégorisation des odeurs ? Considérons les exemples suivants, construits sur le modèle précédent de lignée des couleurs :

Odeur : odorant $>$ floral $>$ jasmin

Odeur : odorant $>$ fruité>citronné

Odeur : odorant $>$ animal $>$ chien mouillé

Odeur : odorant>épicé>poivré

Odeur : odorant $>$ végétal $>$ foin

Ces catégories olfactives ne sont pas de même nature que celles que nous venons d'évoquer dans le registre visuel. Alors que, comme nous l'avons vu, le vermillon appartient nécessairement au « rouge » qui appartient nécessairement au " coloré », dans le cours d'une expérience olfactive le jasmin n'appartient pas nécessairement au floral. Il est tout à fait possible d'identifier dans un cuir une note de jasmin, bien que cette matière relève de la catégorie animale. On peut faire la même remarque pour les descripteurs "fruité ", " animal ", " épicé », "végétal » en regard du niveau super-ordonné. Un trait de ces organisations catégorielles est que les termes les plus spécifiques, loin d'appartenir exclusivement à une seule lignée verticale, qui définirait de ce fait, de manière horizontale, un paysage olfactif bien discriminé, relèvent fréquemment de lignées différentes, sans lien apparent entre elles si on s'en tient aux critères proprement olfactifs.

Il n'est dès lors pas possible d'inférer la catégorie du floral ou du fruité ou de l'animal, etc. depuis un niveau subordonné en l'absence de précision sur le contexte d'énonciation. Autrement dit, le jugement catégoriel des odeurs tend à s'inscrire simultanément dans deux logiques classificatoires, la première strictement olfactive, la seconde renvoyant à des entités associées, qu'il s'agisse de la source de l'odeur, d'un souvenir, du contexte physique et social et, notamment, des normes qui encadrent les jugements perceptifs (Largey \& Watson 1972 ; Corbin 1982 ; Albert 2007), de l'effet produit sur la personne ou d'un peu tout cela à la fois. Pour cette raison, les dimensions verticale et horizontale de la catégorisation des odeurs sont beaucoup plus difficiles à distinguer que dans le registre des couleurs. Si on peut théoriquement concevoir une situation dans laquelle un individu catégorise des odeurs en l'absence de tout référentiel contextuel comme le proposent les protocoles expérimentaux en analyse sensorielle -, nos propres données ethnographiques nous amènent à penser que, en contexte naturel, les jugements catégoriels sont d'une autre nature. Selon notre hypothèse, qui appelle de nouvelles recherches, ils reposent sur la mise en œuvre de comparaisons locales à partir d'inférences complexes, engagées à l'échelle des différentes 
entités évoquées supra et assimilables à des courts-circuits perceptifs entre les lignées verticales et horizontales.

\subsection{Exploitation de la structure du monde perçu}

Selon ce principe, rappelons-le, c'est la combinaison d'attributs d'un même objet de perception qui est explorée. À notre sens, il n'entre pas en ligne de compte lorsque l'objet à percevoir est une couleur ou une odeur. Dans ce cas, odeurs et couleurs sont perçues comme un tout. Certes, la perception d'une couleur, quelle qu'elle soit, joue un rôle important dans les phénomènes de congruence sensorielle, comme cela est bien connu avec l'effet Stroop (Jensen \& Rohwer 1966), mais aussi dans de très nombreux domaines de la vie quotidienne (Sherman \& Clore 2009). Par exemple, si nous rencontrons un cygne noir, l'objet de la perception sera probablement rangé sans trop de difficulté dans la catégorie " oiseaux », mais plus difficilement dans la catégorie " cygne », parce que la combinaison d'attributs « bec+plumes+aile+long cou+noir» est inattendue. Cependant, la catégorisation de la couleur « noir » ne sera nullement perturbée. Il en va de même avec les odeurs. Un Européen, mis pour la première fois en présence d'un durian, aura certainement du mal à ranger ce végétal dans la catégorie " fruits » car la combinaison d'attributs « fruit+odeur excrémentielle » est inattendue, mais ici encore cela ne perturbera pas significativement ${ }^{7}$ la catégorisation de cette odeur. Par conséquent, dans le registre des odeurs, comme dans celui des couleurs, il semble bien que le principe d'exploitation du monde perçu n'ait aucun caractère opératoire sur leur catégorisation lorsqu'elles sont prises isolément. Bien entendu, il est légitime de se demander si, nonobstant l'absence de conséquence sur la pertinence de la notion de catégorie, ce principe existe ou non dans le domaine des odeurs. Intuitivement, compte tenu du caractère holistique de la perception olfactive (Perchec 1999), nous sommes enclins à répondre par la négative. En langage naturel et hors contexte professionnel (parfumeurs, œnologues), rares, en effet, sont les descriptions d'odeurs qui procèdent d'un "découpage » de la forme perçue en unités discrètes. Lorsqu'elles ont lieu, comme dans cette description sur l'Internet de l'odeur des enfants : " Odeur de bébé = couches sales+lait caillé+Mytosil. Hum. » (femme, 47 ans, Haute-Vienne), elles témoignent plus d'une connaissance d'un processus d'agrégation catégoriel que d'une analyse sensorielle à proprement parler. Nous avons également montré (Candau 2002) qu'une exploitation de la structure du monde perçu pouvait exister lorsque la tâche cognitive consiste à catégoriser des odeurs différentes qui se succèdent chronologiquement - avec des attentes, déjouées ou non, de ce que nous avons appelé la " mélodie olfactive » - ou qui se présentent simultanément, avec des attentes, déjouées ou non, de ce que nous avons appelé

7. Si le durian fait l'objet d'une consommation courante en Asie du Sud-Est où il est très prisé, il est à ce jour pratiquement inconnu en France et aux USA où son odeur est parfois catégorisée hors du domaine « fruit », allant de l'ail au pétrole. En France, il occupe ainsi une position intermédiaire entre les catégories «fruit » et « fleur », tandis qu' aux USA, il occupe une position centrale au sein de la catégorie « fleur ». Voir Chrea, Valentin \& Abdi (2009). 
$l^{\prime}$ « harmonie olfactive ». Les exemples culinaires évoqués supra (2.1) illustrent ce processus, où l'enchaînement des attentes naît de la recherche d'indices olfactifs jugés sur la base d'un critère de pertinence à chaque fois renouvelé au fil du cheminement perceptif.

\subsection{Parcimonie linguistique}

Rappelons, en premier lieu, que toutes les odeurs ne sont pas perçues et, parmi celles qui le sont, toutes ne sont pas nommées. Beaucoup, en effet, restent à un niveau infraverbal, pour deux raisons principales. La première est de nature neurophysiologique : nous savons traiter bien plus d'informations par les voies sensorielles que par les facultés cognitives supérieures. Selon le neurobiologiste A. Holley, cela est particulièrement vrai dans le domaine de l'olfaction :

Il est, après tout, compréhensible, dit-il, que la langue n'ait pu individualiser par un terme spécifique chacune des sensations que fournit le monde olfactif, parce qu'il y en a beaucoup trop. (1999: 25)

Cependant, il n'y a là rien de spécifique aux odeurs :

Nous pouvons discriminer plusieurs millions de nuances de couleur, alors que nous ne possédons au mieux que quelques centaines de concepts de couleur. (Pacherie, $2003: 266)$

La seconde raison tient à la spécificité du lexique olfactif : nommer, c'est déjà abstraire l'odeur de l'expérience primordiale et cette abstraction ne va pas de soi quand les outils conceptuels font défaut. En effet, la catégorisation et la mise en mémoire des sensations olfactives ne sont pas facilitées par un lexique stabilisé comme il en existe pour décrire les couleurs. L'évocation d'une odeur s'ancre alors parfois dans l'expérience vécue, reposant sur une comparaison entre « effets » et non plus entre qualités. "Et c'était un parfum qui sentait, j'avais adoré, il sentait frais! Il sentait vraiment le frais, comme un peu... comme quand tu croques dans une pomme, un peu! » (Fanny, 28 ans, Nice). Les échecs cognitifs sont alors fréquents (le "tip of the nose phenomenon", équivalent, en olfaction, du mot sur le bout de la langue), raison pour laquelle on a pu définir à tort l'odorat comme un sens muet.

À tort car, dans le langage naturel, l'idiome des fluides, selon l'expression de J.-K. Huysmans, bien qu'imprécis (mais pas arbitraire ${ }^{8}$ ), est métaphorique voire poétique, ce qui a conduit $\mathrm{D}$. Sperber à voir dans les odeurs « des symboles par excellence » (1974: 130). Un œnologue, par exemple, décrit ainsi le Braquet, un cépage d'appellation Bellet, dans le département des Alpes-Maritimes, dont l'odeur iodée est très typée : c'est l'odeur, dit-il, "d'une petite crique au bord de la Méditerranée, en été quand il fait un peu chaud; des algues sont venues sur la plage, elles commencent à s'abîmer, à fermenter, à se pourrir petit à petit ». Une employée mézoise

8. De même qu'il est tout à fait improbable de trouver le mot rêche associé au toucher de la soie, il est contre-intuitif d'imaginer que l'épithète épais puisse qualifier le parfum de la violette. 
des égouts décrit de manière tout aussi prolixe l'odeur des boues asséchées à l'issue de leur traitement en station d'épuration : "c'est une odeur de champ de vache ou de carnaval, plus exactement les maquillages que l'on me mettait sur le visage pour le carnaval de Frontignan quand j'étais petite », affirme-t-elle ${ }^{9}$. On est ici bien loin de la parcimonie linguistique qui caractérise la catégorisation au niveau de base, si l'on admet que l'on se situe à ce niveau de description dans les deux exemples que nous venons de donner. Du fait de cette absence de parcimonie, le langage naturel des odeurs peut difficilement assurer le rôle de garde-fou catégoriel que rend possible un lexique restreint et précis.

\section{CONCLUSION}

De nos données ethnographiques, il ressort que les « catégories » olfactives ne sont pas des catégories du point de vue de la théorie classique et encore moins du point de vue de la théorie des prototypes. Il paraît tout aussi difficile d'identifier un meilleur exemplaire que des lignées sémantiques avec des relations d'implication entre propriétés, le principe d'économie cognitive se dérobe dès que les odeurs sont décrites à partir de leur source, $c^{\prime}$ est-à-dire la plupart du temps, et la parcimonie linguistique fait le plus souvent défaut. Bien plus, selon les cas, la catégorisation semble s'appliquer à la source odorante, dans d'autres aux molécules qui en émanent, dans d'autres encore à l'événement mental que constitue l'odeur, l'opération cognitive subissant de plus un effet massif du contexte et des divers jugements perceptifs dans lesquels les acteurs sont engagés, si bien que l'on a le sentiment d'être en présence d'un processus quasi stochastique. Ainsi, dans le langage naturel, nous catégorisons les odeurs qui produisent un effet, notamment en termes d'intensité ou de valence hédonique ; celles dont on peut identifier la source ; celles que l'on connaît, ou qui nous sont familières ; celles que l'on ne connaît pas; celles que nous jugeons utiles; celles qui ont une propriété intrinsèque identifiable, etc. Pour cette raison, on pourrait soutenir, en filant la métaphore, que loin d'être roschiennes, elles sont plutôt à forte tendance borgésienne. On sait qu'un texte de Borges évoque « une certaine encyclopédie chinoise » appelée " Empire Céleste du Savoir Éclairé » où il est écrit que les animaux se divisent (on pourrait dire : sont catégorisés) en :

a) appartenant à l'Empereur, b) embaumés, c) apprivoisés, d) cochons de lait,

e) sirènes, f) fabuleux, g) chiens en liberté, $h$ ) inclus dans la présente classification, i) qui s'agitent comme des fous, $\mathrm{j}$ ) innombrables, $\mathrm{k}$ ) dessinés avec un pinceau très fin en poils de chameau, l) et cætera, $\mathrm{m}$ ) qui viennent de casser la cruche, $\mathrm{n}$ ) qui de loin ressemblent à des mouches. (Borges, $1989: 86$ )

De manière similaire, la catégorisation des odeurs ne semble obéir à aucune des logiques mises en avant par la théorie roschienne. Les " catégories » d'odeurs sont des pseudo-catégories ou, au mieux, comme le suggère A. Holley (1997 : 26),

9. Extrait d'un entretien mené par notre collègue A. Jeanjean (cf. Jeanjean 2006). 
des « catégories peu rigides sur lesquels le consensus des locuteurs est rarement acquis », du moins en contexte naturel ${ }^{10}$. Nous associons cette multiplication des axes de catégorisation à la prévalence de la diversité des expériences sur l'expression de catégories sémantiques fortement partagées. Ce "relâchement » catégoriel n'implique pas l'abandon de la quête d'invariants. Les données ethnographiques et expérimentales, que nous avons rassemblées sur les expériences olfactives, nous conduisent à penser qu'il faut tenter de les débusquer dans la structure de l'expérience socio-perceptive - y compris dans ses aspects multisensoriels (Candau 2007, 2010 ; Morrot, Brochet \& Dubourdieu 2001) -, et non dans les seuls effets physiologiques comme cela a été fait dans le domaine de la vision en général et des couleurs en particulier. À cet égard, le traitement analytique de données ethnographiques relatives à des expériences olfactives que nous avons risqué ici, consistant à prendre appui sur la description des jugements perceptifs en lien avec des activités précises, peut constituer une base utile pour une nouvelle mise à l'épreuve des thèses roschiennes appliquées à l'ensemble des formes sensibles (Dubois 2000).

\section{Références}

ALBERT J.-P. (2007), "L'odeur des autres. À propos de quelques stéréotypes ", in G. Boëtsch, C. Hervé \& J. Rozenberg (éds), Corps normalisé, corps stigmatisé, corps racialisé, Bruxelles : De Boeck Université, 77-86.

BAccino T. et al. (2010), "Sharing an Olfactory Experience: The Impact of Oral Communication", Food Quality and Preference 21 (5), 443-452.

Barsalou W. (1983), “Ad-hoc Categories”, Memory and Cognition 11, 211-227.

BARSALOU W. (1985), “Ideals, Central Tendency and Frequency of Instantiation as Determinants of Graded Structure", Journal of Experimental Psychology: Learning, Memory and Cognition 11, 629-654.

BerLIN B. \& KAY P. (1991), Basic Color Terms. Their Universality and Evolution, Berkeley: University of California Press.

BoIsson C. (1997), "La dénomination des odeurs : variations et régularités linguistiques ", Intellectica 24, 29-49.

Borges J.-L. (1989), Obras Completas : El idioma analítico de John Wilkins, Barcelone : Emecé Editores.

Bradford M. \& Caramazza A. (2009), "Concepts and Categories: A Cognitive Neuropsychological Perspective", Annual Review of Psychology 60, 27-51.

CAndau J. (2000), Mémoire et expériences olfactives. Anthropologie d'un savoir-faire sensoriel,

Paris : Presses Universitaires de France.

CANDAu J. (2002), "De la prégnance et de la pertinence olfactive ", in J. Candau, M.-C. Grasse \& A. Holley (éds), Fragrances : du désir au plaisir olfactif, Marseille : Jeanne Lafitte, 65-75.

10. Il en va différemment chez certains professionnels des odeurs (par exemple, les parfumeurs ou les œnologues) où de puissants dispositifs culturels - parmi lesquels un langage, des institutions d'apprentissage et un intérêt partagé pour des objets de même nature physico-chimique - encadrent et normalisent les expériences perceptives et leur communication. 
CANDAU J. (2007), "C'est notre être en entier qui sent. Frontières olfactives et transmodularité de l'esprit ", Cultures \& sociétés 2, 37-43.

CANDAu J. (2010), "Intersensorialité humaine et cognition sociale ", Communications 86, 25-36.

CANDAu J. \& JeAnJean A. (2006), " Des odeurs à ne pas regarder ", Terrain 47, 51-68.

CHAUVEIX S. \& EgOROFF C. (2003), " Mise au point d'une liste de descripteurs sensoriels : une nouvelle approche basée sur la catégorisation ", Psychologie française 48 (4), 79-89.

Chrea C., Valentin D. \& ABdi H. (2009), “Graded Structure in Odour Categories: A Cross-Cultural Case Study", Perception 38 (2), 292-309.

CoRBIN A. (1982), Le miasme et la jonquille. L'odorat et l'imaginaire social auX XVIII $-X I X X^{e}$ siècles, Paris : Aubier Montaigne.

DAVID S. (1997), "Représentations sensorielles et marques de la personne : contraste entre olfaction et audition ", in D. Dubois (éd.), Catégorisation et cognition : de la perception au discours, Paris : Kimé, 211-242.

DAvidoff J. (2001), “Language and Perceptual Categorisation”, Trends in Cognitive Sciences 5 (9), 382-387.

Davidoff J., Davies I. \& Roberson D. (1999), "Colour Categories in a Stone-Age Tribe”, Nature 398, 203-204.

Dubols D. (2000), "Categories as Acts of Meaning: The Case of Categories in Olfaction and Audition”, Cognitive Science Quarterly 1, 35-68.

GiBson J. (1979), The Ecological Approach to Visual Perception, Boston: Houghton Mifflin.

Holley A. (1997), "Le physiologiste et la catégorisation des odeurs ", Intellectica 24, 21-27.

Holley A. (1999), Éloge de l'odorat, Paris : Odile Jacob.

HuYSMANS J.-K. (1977), À rebours, Paris : Gallimard.

JeAnjean A. (2006), Basses CEuvres. Une ethnologie du travail dans les égouts, Paris : CTHS.

Jensen A. \& Rohwer W. (1966), “The Stroop Color. World Test: A Review”, Acta Psychologica 25, 36-93.

KAY P. \& REGIER T. (2003), "Resolving the Question of Color Naming Universals", PNAS 100 (15), 9085-9089.

KAY P. \& REgIER T. (2006), “Language Thought and Color: Recent Developments”, Trends in Cognitive Sciences 10 (2), 51-54.

KAY P. \& Regier T. (2007), “Color Naming Universals: The Case of Berinmo”, Cognition 102 (2), 289-298.

KLEIBER G. (1991), "Hiérarchie lexicale : catégorisation sémantique verticale et termes de base ", Sémiotiques 1 (1), 35-57.

LAKOFF G. \& JoHnSON M. (1985), Les métaphores dans la vie quotidienne, Paris : Éditions de Minuit.

LARGEY G. \& WATSON R. (1972), “The Sociology of Odors", American Journal of Sociology 77 (6), 1021-1034.

LÉVI-Strauss C. (1955), Tristes tropiques, Paris : Plon.

MACÉ M. et al. (2009), “The Time-Course of Visual Categorizations: You Spot the Animal Faster than the Bird", PLoS ONE 4 (6). [en ligne : www.plosone.org/article/info:doi/10.1371/ journal.pone.0005927]

Morrot G., Brochet F. \& Dubourdieu D. (2001), "The Color of Odors", Brain and Language 79 (2), 309-320. 
MURPHY G. \& BRoWNeLl H. (1985), "Category Differentiation in Object Recognition: Typicality Constraints on their Basic Category Advantage", Journal of Experimental Psychology: Learning, Memory, Cognition 11 (1), 70-84.

NAGEL T. (1974), "What is it Like to be a Bat", The Philosophical Review 83 (4), 433-450.

PACHERIE E. (2003), "Modes de structuration des contenus perceptifs visuels ", in J. Bouveresse \& J.-J. Rosat (éds), Philosophies de la perception. Phénoménologie, grammaire et sciences cognitives, Paris : Odile Jacob, 263-289.

Peelen M., Fel-Fel L. \& Kastner S. (2009), “Neural Mechanisms of Rapid Scene Categorization in the Human Visual Cortex", Nature 460, 94-97.

PeRCHEC C. (1999), "Les modèles de la mémoire : revue des études sur l'olfaction et proposition d'un modèle de la mémoire olfactive ", Informations sur les Sciences Sociales 38 (3), 443462.

Proust M. (1987), À la recherche du temps perdu. Du côté du chez Swann, Paris : Robert Laffont.

QUIAN QUIROGA R. et al. (2005), “Invariant Visual Representation by Single Neurons in the Human Brain", Nature 435, 1102-1107.

Regier T., KAY P. \& Cook R. (2005), “Focal Colors are Universal After All”, PNAS 102 (23), 8386-8391.

Regier T., Kay P. \& Khetarpal N. (2007), “Color Naming Reflects Optimal Partitions of Color Space”, PNAS 104 (4), 1436-1441.

Rosch E. (1973), “Natural Categories”, Cognitive Psychology 4 (3), 328-350.

Rosch E. (1975), “Cognitive Representations of Semantic Categories”, Journal of Experimental Psychology 104 (3), 192-233.

SEARLE J. (1995), La construction de la réalité sociale, Paris : Gallimard.

SEARLE J. (2004), Mind. A Brief Introduction, Oxford: Oxford University Press.

Sherman G. \& Clore G. (2009), "White and Black Are Perceptual Symbols of Moral Purity and Pollution", Psychological Science 20 (8), 1019-1025.

SPERBER D. (1974), Le symbolisme en général, Paris : Hermann.

VARELA F., Thompson E. \& Rosch E. (1993), L'inscription corporelle de l'esprit. Sciences cognitives et expériences humaines, Paris : Le Seuil.

WATHELET O. (2009), Anthropologie de la transmission des savoirs et savoir-faire sensoriels. Étude de cas : la transmission familiale d'un patrimoine olfactif, Thèse de doctorat en anthropologie, Nice : Université de Nice-Sophia Antipolis.

WierzBICKA A. (2008), "Why There Are no <Colour Universals> in Language and Thought”, Journal of the Royal Anthropological Institute 14 (2), 407-423.

ZHOU K. et al. (2010), “Newly trained lexical categories produce lateralized categorical perception of color", PNAS 107 (22), 9974-9978. 